\title{
COVID-19 in children and young people
}

\author{
By Matthew D. Snape ${ }^{1}$ and Russell M. Viner ${ }^{2}$ \\ 1Oxford Vaccine Group, Centre for Clinical Vaccinology and Tropical Medicine, Churchill Hospital, Oxford, UK. ²Great Ormond Street Institute for Child Health, University \\ College London, London, UK.
}

Email: matthew.snape@paediatrics.ox.ac.uk; r.viner@ucl.ac.uk

\section{Children have a low risk of COVID-19 and are disproportionately harmed by precautions}

The severe acute respiratory syndrome coronavirus 2 (SARS$\mathrm{CoV}-2$ ) pandemic has brought distinct challenges to the care of children and adolescents globally. Unusually for a respiratory viral infection, children and adolescents are at much lower risk from symptomatic coronavirus disease 2019 (COVID-19) than any other age group. The near-global closure of schools in response to the pandemic reflected the reasonable expectation from previous respiratory virus outbreaks that children would be a key component of the transmission chain. However, emerging evidence suggests that this is most likely not the case. A minority of children experience a postinfectious inflammatory syndrome, the pathology and long-term outcomes of which are poorly understood. However, relative to their risk of contracting disease, children and adolescents have been disproportionately affected by lockdown measures, and advocates of child health need to ensure that children's rights to health and social care, mental health support, and education are protected throughout subsequent pandemic waves.

Evidence from contact-tracing studies suggest that children and teenagers are less susceptible to SARS-CoV-2 infection than adults; however, community swabbing and seroprevalence studies conducted outside of outbreak settings suggest that infection rates are similar to those in older age groups (1-3). Only half of children and teenagers with antibodies against SARS-CoV-2 have experienced symptoms, and there is growing evidence that there is a broad range of presentations, emphasizing the limitations of communitybased prevalence studies based on testing only children with respiratory symptoms. Hospitalization for severe acute COVID-19 in children is rare, but among these pediatric inpatients, respiratory symptoms are more apparent than in infected children in the community (4). Case fatality in hospitalized children is, fortunately, relatively low at $1 \%$ (compared with $27 \%$ across all ages) (4).

The reason for the lower burden of symptomatic disease in children is not yet clear. Upper airway expression of angiotensin-converting enzyme 2 (ACE2), a receptor for the SARS$\mathrm{CoV}-2$ spike protein, increases with age, and higher ACE2 expression correlates with being positive for SARS-CoV-2 genomic RNA in swabs of upper respiratory tracts from symptomatic children, but not with viral load (5). An alternative proposal is the absence in children of maladaptive immune responses that lead to acute respiratory distress syndrome (ARDS) in older age groups (6), but there are likely other unidentified mechanisms.

Understanding the nature of immune responses in children is important given the rare, but potentially severe, multisystem inflammatory syndrome observed in more than 1000 children and adolescents in multiple countries during the first wave of COVID-19 (7). Known variously as pediatric inflammatory multisystem syndrome temporally associated with SARS-CoV-2 (PIMS-TS), multisystem inflammatory syndrome in children (MIS-C), or Kawasaki-like disease, the illness presents with persistent fever accompanied, to a variable extent, by gastrointestinal symptoms, rash, and conjunctival inflammation. Laboratory markers of inflammation are very high, and myocarditis is a distinct, and potentially fatal, feature. Children and young people with PIMS-TS are more likely to have antibodies to SARS-CoV-2 than evidence of virus from nasal swabs, with presentations usually 4 to 6 weeks after infection. The cardiac involvement initially led to this condition being described as a variant of Kawasaki disease (in which an unknown trigger leads to an inflammatory disease, resulting in coronary artery inflammation). However, a comprehensive case series clearly delineated PIMS-TS from Kawasaki disease, with children who experience PIMS-TS being substantially older and with increased circulating concentrations of ferritin (a marker of inflammation) and D-dimer and troponin (markers of cardiovascular damage), which are rarely seen in Kawasaki disease (8). A dominant feature of PIMS-TS is myocarditis, transient myocardial dysfunction, and shock, which are present in approximately half of UK and U.S. case series $(8,9)$.

In the UK, a Delphi national consensus statement has recently been proposed (10) to guide investigation and management of this condition, which focuses on supportive care and enrolling patients into a specific arm of the RECOVERY randomized controlled trial to evaluate the use of corticosteroids and intravenous immunoglobulin in patients with acute PIMS-TS. Fortunately, fatalities are rare [occurring in 10 of the 570 cases reported to the U.S. Centers for Disease Control and Prevention between March and July 2020, and none of 52 cases in a UK series $(4,9)]$. However, the long-term 
consequences are unknown, and all children and teenagers who experience PIMS-TS require ongoing cardiac review. Proposed mechanisms for this illness have focused on a maladaptive acquired immune response to SARS-CoV-2 infection, and a dysregulated humoral immune response is suggested by increased antibodies against multiple, nonSARS-CoV-2, respiratory viruses in severe MIS-C but not mild MIS-C or acute COVID-19 (5).

Understanding this response is crucial when considering the risks and benefits of immunizing children against COVID-19, should a vaccine become available. Of the vaccines being tested in clinical trials, none have yet been administered to children, with priority instead being given, appropriately, to older age groups. The most advanced candidate, ChAdOX1-nCOV-19, is currently in phase 2 and 3 studies that include a pediatric arm for 5 to 12 year olds (NCT04400838), receiving half the full adult dose in this study. However, this study arm is not currently active and will commence enrolment only once the safety profile in adults is more complete. Given the low rates of disease in children, they are likely to be a low priority to receive a vaccine unless it is definitively shown that (i) children have an important role in the transmission of the virus and (ii) the vaccine reduces viral shedding (and hence reduces transmission).

To what extent do children transmit SARS-CoV-2? Recent reports that young children acutely unwell with COVID-19 have concentrations of viral RNA in nasal aspirates that are similar to, or higher, than adults (5) raised concerns that their role in transmission may have been underestimated. However, one of these studies compared children within the first week of illness with adults with more than 7 days of symptoms, when viral load is expected to be reduced (5). Such studies need to be interpreted with consideration of the very low numbers of children with symptomatic COVID-19.

Of greater concern is the possibility that viral shedding could be occurring from asymptomatic children and that, given schools" "bridge" households, this could create a pool of ongoing viral circulation responsible for introductions of virus to the pupils' homes and beyond. Understanding this issue is fundamental to resolving what has been an unprecedented global disruption to primary (children of $\sim 5$ to 11 years) and secondary (children aged 11 to 18 years) education. Given the near universal closing of schools in conjunction with other lockdown measures, it has been difficult to determine what benefit, if any, closing schools has over other interventions. However, there is some reassurance: Multiple studies of contacts of primary and secondary school children with known SARS-CoV-2 infection showed minimal onward transmission in schools (3). Furthermore, after the reopening of primary schools in the UK, only 1 of 23,358 nasal swabs taken from children in June 2020 had detectable SARS-CoV2, giving an estimate of 3.9 cases per 100,000 students (2).
Looked at from another perspective, when household outbreaks of infection have occurred, it appears that children were responsible for only a small minority of household introductions of the virus. Also, recent surveys found that reopening of schools in a number of European countries in April and May had no clear impact on community transmission, with cases continuing to fall in most countries after reopening (11).

Nevertheless, recent experiences of substantial outbreaks of COVID-19 related to children and teenagers show that there is no room for complacency. In May, an Israeli secondary school was shut shortly after a postlockdown reopening after the identification of two symptomatic students independently infected with SARS-CoV-2. A subsequent schoolwide testing campaign revealed that $153(13.2 \%)$ students and $25(16.6 \%)$ staff had detectable SARS-CoV-2 infection, and contact tracing revealed a further 87 cases in non-school attendees (12). Although formal studies were not conducted to definitively show school-based transmission, potential contributory factors included a heat wave that led to extensive use of air-conditioning and exemptions from face mask wearing, relatively crowded classrooms (with 35 to 38 per class with 1.1 to $1.3 \mathrm{~m} 2$ between students), and shared schoolyard and outdoor spaces.

As schools in the Northern Hemisphere reopen after summer holidays, risk mitigation strategies adopted to variable degrees include creating separate cohorts (or "bubbles") within schools that interact minimally with each other, use of face masks in crowded areas (if not the classroom itself), and regular screening of students and staff. The coming months will provide an invaluable opportunity to identify which of these measures are most effective at minimizing transmission, to generate a standard "best practice" that balances young peoples' rights to an education with the need to protect the broader community from further transmission. However, it is inevitable that there will be students attending school while infected with SARS-CoV-2, and likely there will be some school outbreaks, with the frequency of these events reflecting levels of community transmission. Regardless, it is hard to support the opening of retail and hospitality sectors while schools remain shut, as occurred in many countries earlier this year.

School closures and attendant loss of other protective systems for children (such as limited social care and health visiting) highlight the indirect, but very real, harms being disproportionately borne by children and teenagers as a result of measures to mitigate the COVID-19 pandemic. In the UK, it is estimated that the impact on education thus far may lead to a quarter of the national workforce having lower skills and attainment for a generation after the mid-2020s, leading to the loss of billions of dollars in national wealth (11). Additionally, there are a variety of other harms to children's 
health, including the risk of reemergence of vaccine-preventable diseases such as measles because of disruptions to immunization programs.

There are many other areas of potential indirect harm to children, including an increase in home injuries (accidental and nonaccidental) when children have been less visible to social protection systems because of lockdowns. In Italy, hospitalizations for accidents at home increased markedly during the COVID-19 lockdown and potentially posed a higher threat to children's health than COVID-19 (13). UK pediatricians report that delay in presentations to hospital or disrupted services contributed to the deaths of equal numbers of children that were reported to have died with SARS-CoV-2 infection (14). Many countries are seeing evidence that mental health in young people has been adversely affected by school closures and lockdowns. For example, preliminary evidence suggests that deaths by suicide of young people under 18 years old increased during lockdown in England (15).

The role of children in transmission of SARS-CoV-2 remains unclear; however, existing evidence points to educational settings playing only a limited role in transmission when mitigation measures are in place, in marked contrast to other respiratory viruses. In the event of seemingly inevitable future waves of COVID-19, there is likely to be further pressures to close schools. There is now an evidence base on which to make decisions, and school closure should be undertaken with trepidation given the indirect harms that they incur. Pandemic mitigation measures that affect children's wellbeing should only happen if evidence exists that they help because there is plenty of evidence that they do harm.

\section{REFERENCES AND NOTES}

1. What's the STORY, "Serum testing of representative youngsters" (2020); https://bit.ly/2H3OaGa.

2. S. Ladhani, PHE publications gateway number: GW-1599 (Public Health England, 2020); https://bit.ly/35G0mVV.

3. R. M. Viner et al., medRxiv 20108126 [Preprint] 21 August 2020; doi:10.1101/2020.05.20.20108126.

4. O. Swann et al., BMJ 370, m3249 (2020). doi:10.1136/bmi.m3249

5. L. M. Yonker et al., J. Pediatr. 10.1016/j.jpeds.2020.08.037 (2020).

6. A. Fialkowskiet al., Pediatr. Pulmonol. 10.1002/ppul.24981 (2020).

7. A. H. Rowley, Nat. Rev. Immunol. 20, 453 (2020). doi:10.1038/s41577-020-0367-5 Medline

8. E. Whittaker et al., JAMA 10.1001/jama.2020.10369 (2020).

9. S. Godfred-Cato et al.; California MIS-C Response Team, MMWR Morb. Mortal. Wkly. Rep. 69, 1074 (2020). doi:10.15585/mmwr.mm6932e2 Medline

10. R. Horwood et al., medRxiv 20156075 [Preprint] 23 July 2020; do: $10.1101 / 2020.07 .17 .20156075$

11. The DELVE Initiative, "Balancing the risks of pupils returning to schools" (Royal Society DELVE Initiative, 2020); https://bitly/3iy805.

12. C. Stein-Zamir et al., Euro Surveill. 10.2807/1560-7917.ES.2020.25.29.2001352 (2020).

13. S. Bressan et al., Arch. Dis. Child. 10.1136/archdischild-2020-319547 (2020).

14. R. M. Lynnet al., Arch. Dis. Child. 10.1136/archdischild-2020-319848 (2020).

15. D. Odd et al., "Child suicide rates during the COVID-19 pandemic in England: Realtime surveillance" (Healthcare Quality Improvement Partnership 2020); https://bitly/3moxo2V.

Published online 21 September 2020

10.1126/science.abd6165 